\title{
Experiences and Challenges While Managing the Inpatient Psychiatry Unit Dedicated for COVID-19 Patients
}

\section{Nahla Mahgoub1, Smita Agarkar1, Michael Radosta'2, Francine Fakih², Brynne Delaney Calleran², Robert Clark ${ }^{2}$, Deborah Cherubin ${ }^{2}$, Faraj Faour ${ }^{2}$, Donna Anthony ${ }^{1}$}

${ }^{1}$ Weill Cornell Medicine/Gracie Square Hospital, New York, USA

${ }^{2}$ Gracie Square Hospital, New York, USA

Email: namd06@aol.com, smd9004@med.cornell.edu, mir9044@nyp.org,frf9029@nyp.org, brc9055@nyp.org, roc9191@nyp.org,dec9124@nyp.org,faf9022@nyp.org,dta2001@nyp.org

How to cite this paper: Mahgoub, N., Agarkar, S., Radosta, M., Fakih, F., Calleran, B.D., Clark, R., Cherubin, D., Faour, F. and Anthony, D. (2021) Experiences and Challenges While Managing the Inpatient Psychiatry Unit Dedicated for COVID-19 Patients. Open Journal of Psychiatry, 11, 12-19.

https://doi.org/10.4236/ojpsych.2021.111002

Received: December 19, 2020

Accepted: January 16, 2021

Published: January 19, 2021

Copyright $\odot 2021$ by author(s) and Scientific Research Publishing Inc. This work is licensed under the Creative Commons Attribution International License (CC BY 4.0).

http://creativecommons.org/licenses/by/4.0/

\begin{abstract}
The COVID-19 pandemic has significantly changed the mental health care. Treating psychiatric patients with COVID-19 poses multiple challenges in the inpatient psychiatric setting in terms of mitigating transmission of the virus. Gracie Square Hospital, a freestanding psychiatric hospital located in New York City, devoted a unit to treating COVID-19 patients requiring inpatient psychiatric treatment. This paper describes our experiences and challenges while managing the psychiatric COVID-19 unit that may serve as a model for other health care facilities during the COVID-19 pandemic.
\end{abstract}

\section{Keywords}

COVID-19 Pandemic, Psychiatric Patients with COVID-19, COVID-19

Psychiatric Unit

\section{Introduction}

Coronavirus 2019 (COVID-19) has become a global public health concern since the illness can vary from asymptomatic infection to severe disease and mortality. On March 11, 2020, COVID-19 was declared a pandemic by the World Health Organization (WHO). On March 13, 2020, a national emergency was declared in the United States concerning the COVID-19 outbreak [1].

New York City was the hardest hit place in the United States and considered as the epicenter of America's COVID-19 crisis. According to the New York City Department of Health and Mental Hygiene, approximately 40,000 individuals 
were infected and 1000 were killed in the city during the first month of the outbreak [2].

The impact of COVID-19 pandemic on New York City hospitals was severe. In response to rapid spread of the COVID-19 virus and increasing number of cases, hospitals' bed capacity was depleted. To help with the surging demand, some facilities converted their psychiatric beds to critical care needs [3].

Gracie Square Hospital is a freestanding psychiatric hospital located on the Upper East Side of Manhattan in New York City and a member of the New York Presbyterian (NYP) Regional Hospital Network which has several inpatient units. The hospital continued to admit and provide care for patients requiring inpatient psychiatric treatment during the outbreak. Concerns were raised related to preventing and managing COVID-19 spread on the inpatient units. In response to this unprecedented situation, the hospital was dedicated the first unit in Manhattan to treating COVID-19 patients in acute psychiatric crisis. Gracie Square Hospital teamed up with NYP leadership to optimize patient care and mitigate the effects of the outbreak at the individual and hospital level.

This paper describes the management and operation of a psychiatric COVID-19 unit that may serve as a model for other health care facilities during the COVID19 pandemic.

\section{The Development of the Psychiatric COVID-19 Unit}

At Gracie Square Hospital, guidance from the Centers for Disease Control and Prevention (CDC) was strictly followed and safety measures were standardized on all units. These include: monitoring the CDC website for updated information, preparing a multidisciplinary team of medicine and psychiatry, attending to the existing infection control guidelines for enhancing hand hygiene and disinfection, applying social distancing strategies to increase space among patients and among staff, screening patients for COVID-19 at time of admission, restricting visitation to limit the potential transmission from visitors, using personal protective equipment (PPE), creating a temperature monitoring protocol for all patients, identifying patients who are at increased risk for severe symptoms from COVID-19 secondary to medical comorbidities, isolating patients with suspected or confirmed COVID-19, and quarantining the close contacts.

While implementing the CDC recommendations, we faced the following unique challenges among psychiatric patients: lack of mental capacity to give consent to treatment, impaired judgment, treatment refusal, uncooperativeness, difficulty complying or comprehending social distancing, aggression, psychosis, and worsening psychiatric symptoms due to COVID-19 outbreak. At times, behavioral dysregulation made it impossible for patients to adhere to isolation precautions. Some patients contravened precautions, for instance, by spitting during agitated episodes or drinking the alcohol-based hand sanitizer.

Accordingly, Gracie Square Hospital converted one of its general psychiatric units into a psychiatric COVID unit. The unit was dedicated entirely to treating 
COVID-19 patients who need psychiatric care with the goal of minimizing the risk of exposure to patients and staff. This conversion process was executed in a timely manner considering the rapidly evolving outbreak. The hospital designated a multidisciplinary team to meeting the complex needs of this unique patient population. Moreover, the department of Infection Prevention and Control (IPC) at NYP was providing ongoing clinical advice including clinical protocols and consultation on individual patient cases.

\section{Operationalizing the Psychiatric COVID-19 Unit}

Bed capacity: the identified unit has bed capacity of 35, with 10 beds inside a separate suite on both sides of the unit. These suites were separated from the main hallway through a door on either side. This enabled us to first isolate COVID-19 patients in two separate suites with a total capacity of 20 beds. Once one suite was filled, we proceeded to fill the other one. It was decided to admit only COVID-19 patients to these suites to avoid exposure to other patients. As the outbreak in New York City worsened and the hospital started getting more COVID-19 patients, we had to then convert the entire 35 beds into a COVID-19 unit.

Source of referral: all COVID-19 patients were referred from the emergency rooms and inpatient medical units of other hospitals in New York City. Moreover, patients who turned positive on a non-COVID-19 unit at our hospital, were transferred to the psychiatric COVID-19 unit. We also received patients from other inpatient psychiatric units which were converted to critical care service.

The PPE use: the unit followed the CDC recommendation and infection control guidelines. All patients were wearing a face mask. Healthcare personnel used disposable face mask, hospital scrub, and gloves as well as reusable face shield.

Admitting process: patients were seen in the admitting suites by medicine and psychiatry for severity of their symptoms. All patients must wear a face mask once they step into the admitting suite. If a COVID-19 patient refuses to wear a mask because of agitation or florid psychosis, emergency medications would be administered to maintain behavioral control and prevent spread of the virus. When transferring a newly admitted patient from the admitting suite to the unit, a security officer must be present along with nursing staff to ensure patient's compliance with the safety measures.

Patient's safety: upon arrival to the unit, patients would be taken immediately to their room and given written information (e.g., card) to reinforce the need for the safety measures (isolation, face mask, hand hygiene). This is in addition to posting visual alerts such as signs throughout the unit and regular overhead announcements to remind the patient of the safety measures. Patients were placed in single rooms with a separate bathroom. Meals were served in the patients' rooms. Equipment designated for the psychiatric COVID-19 unit such as medication cart and vital sign machines would not be used elsewhere in the hospital.

Patient's assessment: all patients were assessed thoroughly for severity of 
COVID-19 symptoms, medical comorbidities, mental capacity to assent to treatment, psychiatric diagnosis, behavior, psychosocial status, and the level of care needed. Risk of suicide was carefully assessed. Recognizing the warning signs of suicide and creating a safety plan were crucial.

Team structure: a multidisciplinary team consists of psychiatry, psychology, medicine, nursing, social worker staff, therapeutic activity therapists, and environmental services established a framework for standardized protocols in collaboration with the hospital leaders. The protocols included the followings: management of medical crisis, criteria for emergency room transfer, criteria for COVID-19 retesting, proper use of PPE, disinfection guidelines, psychopharmacological management, behavioral interventions, response to psychiatric emergency, and discharge planning. The chief of the psychiatric COVID-19 unit was in charge of the operations and coordinated the implementation of the administrative and clinical aspects of these protocols. The treating team met with hospital leaders on a daily basis through web conference to discuss challenges and to update the protocols as clinically necessary.

Staff safety: the team also established a protocol to ensure staff protection as per the CDC recommendations. This included educating staff on the virus transmission, conducting the meetings through web conferencing, separating workstations to maintain social distancing, maximizing the availability of the PPE and hand sanitizer, training about safe PPE practice, designating a space for donning and doffing procedure to avoid contact with potentially contaminated PPE, disinfecting all supplies such as blood pressure machines as well as desktops, testing health care workers, and developing a return-to-work policy.

Staff psychological well-being: hospital leaders opened a transparent dialogue with the entire staff and responded to their concerns regarding the uncertainty of the situation. Reassurance, guidance, and debriefing were provided daily to ease the burdens. The unit maintained adequate staffing to ensure safety and limit stress. The hospital also provided free COVID-19 testing to the staff, free meals, free parking, and other financial supports to those in need. Staff was also offered individual counseling to help manage fears of infection.

Medical management: in addition to the regular daily psychiatric assessment, patients were evaluated by internal medicine working on the unit on a daily basis and as clinically indicated. An infectious disease team was available for consultation. Nursing staff monitored respiratory symptoms and vital signs including temperature and oxygen saturation as per protocols. Moreover, a medical team was available twenty-four hours a day for urgent medical care and possible transfer to the emergency room for acute decompensation.

Psychiatric management: worsening of psychiatric symptoms such as paranoia, anxiety, and depression during the COVID-19 outbreak was considered and addressed through optimizing the medication management. Individual therapy sessions were provided by the unit psychologist maintaining social distancing to address patients' fear of COVID-19 pandemic. If 1:1 observation was needed for an acutely suicidal patient, observing staff rotated every hour, main- 
tained social distancing and wore PPE.

Therapeutic activities. all therapeutic groups were suspended because of physical contact. The unit psychologist and therapeutic activity therapists created a modified behavioral plan for therapeutic activities that was done inside the patient's room such as art therapy. Patients were given a box of supplies including markers, art work, puzzles and journals to use in their rooms. Supplies would be discarded after discharge. Moreover, the hospital rapidly purchased and implemented technology to assist treatment. Each patient was supplied with an iPad and MP3 player for music.

Medication administration: the unit prohibited patients from lining up for medications. Patients remained in their rooms during medication administration. When appropriate, one patient at a time is allowed to come to the medication room and then to go back directly to his room. Proper disinfection was implemented at the medication window between each patient.

Managing medication refusal: when a COVID-19 patient is incapable of giving consent to treatment and refusing psychiatric medications, the treating psychiatrist submits a request to obtain court permission to administer medications over his objection. With the help of the Governor and swift action taken to relax legislation, the hospital conducted remote court appearances (Tele-Court) in order to minimize risk of exposure during transporting the patients.

Response to psychiatric emergency. a PPE Go-Bag was developed to assist in expediting responses to emergencies. We utilized the seclusion room when a COVID-19 patient becomes agitated. We did not seclude patients in their rooms as they could not be observed. When a COVID-19 patient refuses safety measures such as isolation or wearing a mask because of psychosis, he is determined to be an imminent danger to others and subsequently, he would be secluded. The seclusion room was disinfected by the environmental services after each use.

Visitation: all visitations were suspended in accordance with state mandates, with patients' agreement and education, in order to reduce risk of transmission from the visitors. The unit provided iPads allowing patients to communicate visually and audibly with their family members. All iPads were disinfected after each use.

Disinfecting the unit the environmental services increased the number of staff and doubled the number of cleaning. Patient rooms, bathrooms, environmental surfaces (e.g., floor, tables, chairs) and staff area (e.g., desks, computers) were disinfected twice a day using an approved hospital disinfectant. Seclusion room, patient-care devices (e.g., blood pressure machines), and patient supplies (e.g., iPads) were disinfecting after each use.

Discharge planning, the unit avoided discharging patients who are still in the isolation period. Patients needed to have two COVID-19 negative tests $24 \mathrm{hr}$ apart in absence of fever or any other COVID-19 symptoms. They could then be transferred to a non-COVID-19 unit or discharged. The approval of the department of IPC is needed before transferring patients to a non-COVID-19 unit. It was also possible to discharge patients to the community if psychiatrically stable. 
Discharge planning was carefully considered particularly if patients are returning home and included family education through phone conferences. In New York City, specific shelters were designated for COVID-19 patients who are medically and psychiatrically stable and needed to be discharged. Social worker staff coordinated the discharge plan including psychiatric and medical aftercare.

\section{Discussion}

The COVID-19 pandemic highlighted the limitations of understanding related to the pathogenesis of this virus and the paucity of publications in the medical literature pertaining to its clinical aspects.

For example, the $\mathrm{CDC}$ and $\mathrm{WHO}$ were constantly updating the view on the COVID-19 mode of transmission. Respiratory droplets and close contact were the known routes of transmission [4]. Some evidence supported airborne transmission [5]. In addition, the virus is detected in samples of gastrointestinal tract, saliva, stool, and urine [6].

The Food and Drug Administration (FDA) approved three COVID-19 diagnostic panels developed by the CDC: molecular test, rapid antigen test, and serology antibody test [7]. The detection of viral nucleic acid is the standard for diagnosis of COVID-19. However, the detection of COVID-19 nucleic acid has high specificity and low sensitivity, thus there may be false-negative results [8].

Evidence suggested that COVID-19 is a multi-systemic infectious disease and its clinical manifestations were not well studied [9]. Until recently, there was no FDA approved antiviral medication or vaccine for COVID-19 thus the treatment is symptomatic to alleviate symptoms and prevent complications. In October 2020, the FDA approved the antiviral drug; remdesivir, for the treatment of COVID-19 patients but should only be administered in a hospital setting capable of providing acute clinical care [10].

The heterogenicity of clinical presentations led to the implementation of various public health measures. For example, in December 2020, the FDA issued emergency use authorization for first COVID vaccine in individuals 16 years of age and older [11]. The Centers for Medicare \& Medicaid Services (CMS) ensured that hospitals have the capacity to handle surge of COVID-19 patients [12]. The CDC applied different public strategies of isolation, quarantine, social distancing and community containment in order to control the outbreak [13].

While CDC and WHO recommended telehealth and outpatient services during the outbreak, inpatient psychiatric care was necessarily required for certain patients. In addition to the recommended measures for all healthcare facilities, special consideration was needed for psychiatric inpatients given the clinical complexities.

In a South Korea psychiatric hospital, COVID-19 quickly spread through a psychiatric unit and infected 101 out of 103 patients during the outbreak. This led to the development of psychiatric COVID-19 units in attempt to contain the virus at the hospital [14]. 
For example, an Israeli psychiatric hospital [15] and the University of Rochester Medical Center [16] created a unit to treat COVID-19 patients who need inpatient psychiatric treatment.

Similarly, Gracie Square Hospital dedicated a unit to the treatment of psychiatric patients with COVID-19. Protocols and safety measures were continuously updated to support rapidly progressing situation. There was a total of 48 psychiatric patients with COVID-19 admitted to our unit during the period of April-May 2020. Thirty-three patients were referred from the emergency rooms and medical floors, and they tested positive for COVID-19 prior to admission. Fifteen patients turned positive during their hospital stay on the non-COVID-19 units of our hospital and they were transferred to the psychiatric COVID-19 unit. The peak census was 26 patients. At time of admission, all patients presented with typical symptoms of fever, cough and sore throat but not medically ill enough to be hospitalized on the medical floor. During the hospital course, ten patients were converted to negative and eight patients were transferred to the emergency room for respiratory complications. Forty patients were discharged to the community. All staff on the psychiatric COVID-19 unit remained negative once the unit was established.

\section{Conclusions}

COVID-19 pandemic has dramatically changed psychiatric practice over a short period of time given the level of clinical uncertainty and unique circumstances. The unprecedented task of preventing and managing COVID-19 transmission on the inpatient psychiatric setting requires consideration of the distinctive challenges encountered within this patient population and the development of contingency plans to address them [17].

Our experience on the psychiatric COVID-19 unit at Gracie Square Hospital underlines the clinical complexity of COVID-19 patients with acute psychiatric illness and the multidimensional approaches that can be applied. These approaches may include the consideration of integrated collaboration, open communication, transparency, patient education, assessment of patients' vulnerability, accessibility to infectious disease consultation, reorganization of clinical activities to maintain safety, expansion of the use of technology, development of a supportive platform to maintain psychological well-being of staff, optimization of safety measures, and standardization of protocols and clinical practice.

We encourage other inpatient psychiatric facilities that cannot develop COVID19 specific units to create a culture of safety and education in an attempt to reduce morbidity and mortality related to the virus while delivering necessary mental health care to these patients.

\section{Declaration}

All authors have no conflicts of interest and did not receive any financial support from any organization regarding the content of this paper. 


\section{References}

[1] https://www.cdc.gov/nchs/data/icd/Announcement-New-ICD-code-for-coronaviru s-3-18-2020.pdf\#: :text=On\%20March\%2011\%2C\%202020,COVID\%2D19\%20Out break

[2] https://www.nytimes.com/interactive/2020/04/01/nyregion/nyc-coronavirus-cases$\underline{\text { map.html }}$

[3] https://www.healthaffairs.org/do/10.1377/hblog20200317.457910/full/

[4] https://www.cdc.gov/coronavirus/2019-ncov/prevent-getting-sick/how-covid-sprea ds.html

[5] Setti, L., Passarini, F. and De Gennaro, G. (2020) Airborne Transmission Route of COVID-19: Why 2 Meters/6 Feet of Inter-Personal Distance Could Not be Enough. International Journal of Environmental Research and Public Health, 17, 2932.

[6] Wang, L., Wang, Y., Ye, D., Liu, Q., et al. (2020) Review of the 2019 Novel Coronavirus (SARS-CoV-2) Based on Current Evidence. International Journal of Antimicrobial Agents, 55, Article No. 105948.

[7] https://www.fda.gov/consumers/consumer-updates/coronavirus-testing-basics\#: :te $\mathrm{xt}=$ Molecular\%20diagnostic\%20tests\%20that\%20detect,all\%20of\%20the\%20time

[8] Tahamtan, A. and Ardebili, A. (2020) Real-Time RT-PCR in COVID-19 Detection: Issues Affecting the Results. Expert Review of Molecular Diagnostics, 20, 453-454.

[9] Temgoua, M.N., Endomba, T.F., Nkeck, J.R., et al. (2020) Coronavirus Disease 2019 (COVID-19) as a Multi-Systemic Disease and its Impact in Low- and Middle-Income Countries (LMICs). SN Comprehensive Clinical Medicine, 2, 1377-1387.

[10] FDA News Release, October 22, 2020.

https://www.fda.gov/news-events/press-announcements/fda-approves-first-treatme nt-covid-19\#: :text=Today\%2C\%20the\%20U.S.\%20Food\%20and,of\%20COVID\%2 D19\%20requiring\%20hospitalization

[11] FDA News Release, December 11, 2020. https://www.fda.gov/news-events/press-announcements/fda-takes-key-action-fightagainst-covid-19-issuing-emergency-use-authorization-first-covid-19

[12] https://www.cms.gov/files/document/covid-hospitals.pdf

[13] https://www.cdc.gov/coronavirus/2019-ncov/prevent-getting-sick/social-distancing. $\underline{\mathrm{html}}$

[14] https://www.medscape.com/viewarticle/930659

[15] https://www.timesofisrael.com/worlds-first-psychiatric-coronavirus-ward-opens-in -israel/

[16] https://catalyst.nejm.org/doi/full/10.1056/CAT.20.0249

[17] Callaghan, A.W., Chard, A.N., Arnold, P., et al. (2020) Screening for SARS-CoV-2 Infection Within a Psychiatric Hospital and Considerations for Limiting Transmission Within Residential Psychiatric Facilities-Wyoming, 2020. Morbidity and Mortality Weekly Report, 69, 825-829. 